PROCEEDINGS OF THE

AMERICAN MATHEMATICAL SOCIETY

Volume 128, Number 3, Pages 885-891

$\mathrm{S}$ 0002-9939(99)05576-8

Article electronically published on October 25, 1999

\title{
YANG INDEX OF THE DELETED PRODUCT
}

\author{
SIMEON T. STEFANOV \\ (Communicated by Thomas Goodwillie)
}

\begin{abstract}
For any $\kappa \geq 1$ a $\kappa$-dimensional polyhedron $Y_{\kappa}$ is constructed such that the Yang index of its deleted product $Y_{\kappa}^{*}$ equals $2 \kappa$. This answers a question of Izydorek and Jaworowski (1995).

For any $\kappa \geq 1$ a $2 \kappa$-dimensional closed manifold $M$ with involution is constructed such that index $M=2 \kappa$, but $M$ can be mapped into a $\kappa$-dimensional polyhedron without antipodal coincidence.
\end{abstract}

The deleted product of $Y$ is the space

$$
Y^{*}=Y^{2} \backslash \Delta,
$$

where $\Delta$ is the diagonal of $Y^{2}$. There is a natural free involution $T(x, y)=(y, x)$ acting in $Y^{*}$.

Our goal is to compute the Yang index of the deleted product of some polyhedra (with respect to the involution $T$ ). In particular, we answer the question in [3] of whether there exists a $\kappa$-dimensional polyhedron $Y_{\kappa}$ with index $Y_{\kappa}^{*}=2 \kappa$. It is shown that the space $Y_{\kappa}=\left[\Delta^{2 \kappa+2}\right]^{\kappa}$ has index $Y_{\kappa}^{*}=2 \kappa$.

In fact, we shall find in $Y_{\kappa}^{*}$ a closed manifold $M_{2 \kappa}$ with index $M_{2 \kappa}=2 \kappa$. Then the projection $p(x, y)=x$ is a map $p: M_{2 \kappa} \rightarrow Y_{\kappa}$ without antipodal coincidence. Other examples of such manifolds (or even polyhedra) are not known to us. Let us note the theorem of Šchepin [4], which asserts that every map $f: S^{2 \kappa} \rightarrow P_{\kappa}$ of the $2 \kappa$-sphere into a $\kappa$-dimensional polyhedron has an antipodal coincidence.

First some notation.

$\Delta^{n}$ is a standard $n$-simplex in $\mathbb{R}^{n}$ with center in the origin $O$.

Let $P$ be a simplicial complex.

For $x \in P,[x]$ denotes the carrier of $x$, i.e. the (closed) simplex containing $x$ in its interior.

If $a \in P$ is a vertex, its $\operatorname{star} \operatorname{St}(a)$ is the union of all open simplexes with vertex $a$.

$[P]^{\kappa}$ denotes the $\kappa$-dimensional skeleton of $P$.

All maps are assumed to be continuous.

Now we shall list some properties of the Yang index that we shall make use of, and refer the reader to [7] for the definition and the whole index theory.

Let $X$ be a compact metric space with a free involution $T: X \rightarrow X$. Then its Yang index is defined inductively by means of the equivariant homology groups with coefficients in $\mathbb{Z}_{2}$. We denote it here by index $X$. An important property of

Received by the editors December 18, 1995 and, in revised form, September 5, 1996.

2000 Mathematics Subject Classification. Primary 55M20.

Key words and phrases. Yang index, deleted product, antipodal coincidence.

(C)1999 American Mathematical Society 
the index is that if index $X \geq n$, then every map $f: X \rightarrow \mathbb{R}^{n}$ has an antipodal coincidence: $f(T x)=f(x)$.

Note also that index $X \leq \operatorname{dim} X$.

The following useful proposition estimates the index of a manifold.

Proposition. Let $M_{n}$ be an n-dimensional closed manifold with a free involution $T: M_{n} \rightarrow M_{n}$. Suppose that there exists an odd map $\varphi: M_{n} \rightarrow S^{n}(i . e . \varphi(T x)=$ $-\varphi(x))$ with $\operatorname{deg}_{2} \varphi=1$, where $\operatorname{deg}_{2}$ is the degree mod 2. Then index $M_{n}=n$.

Proof. Let $z^{n}$ be the invariant fundamental cycle $\bmod 2$ in $M_{n}$. Then $\varphi_{*}\left(\left[z^{n}\right]\right) \neq 0$ (in the Cech homologies mod 2). We have $\nu\left(\left[z^{n}\right]\right)=\nu\left(\varphi_{*}\left[z^{n}\right]\right) \neq 0$, as follows from the properties of the index homomorphism $\nu$ (cf. [7] for the definition of $\nu$ ). But this means that index $M_{n} \geq n$ by definition. The converse inequality follows from the fact that index $\leq \operatorname{dim}$.

\section{The MAIN THEOREMS}

Let $M$ be a finite set in $\mathbb{R}^{n}$ and let $\sigma_{1}^{\kappa}, \sigma_{2}^{\kappa}$ be two $\kappa$-dimensional simplexes with vertices in $M$, without a common vertex. Suppose that every two such simplexes either do not intersect or have a single common point, interior to both $\sigma_{1}^{\kappa}$ and $\sigma_{2}^{\kappa}$.

We shall denote by $\#_{\kappa}(M)$ the number of intersections of pairs $\left\{\sigma_{1}^{\kappa}, \sigma_{2}^{\kappa}\right\}$ as above. Such an intersection will be called a $\kappa$-intersection.

For example, if $M=\{5$ points lying on a circle $\}$, then $\#_{1}(M)=5$.

Lemma 1. There exists in $\mathbb{R}^{2 \kappa}$ a set $M$ of $2 \kappa+3$ points such that $\#_{\kappa}(M)=1$.

The proof is given in Section III. For example, in the case $\kappa=1$ it suffices to take 5 points in $\mathbb{R}^{2}$ in general position, whose convex hull is a triangle.

Consider now the complex

$$
Y_{\kappa}=\left[\Delta^{2 \kappa+2}\right]^{\kappa} .
$$

We shall prove that index $Y_{\kappa}^{*}=2 \kappa$. Set

$$
M_{2 \kappa}=\left\{(x, y) \in Y_{\kappa}^{2} \mid[x] \cap[y]=\varnothing\right\} .
$$

Clearly, $M_{2 \kappa}$ is an invariant compact subset of $Y_{\kappa}^{*}$.

Lemma 2. $M_{2 \kappa}$ is a closed manifold.

The proof of this interesting proposition is given in Section II. Notice that $M_{2 \kappa}$ has a structure of a cell complex. It is also easy to show that there is a deformation of $Y_{\kappa}^{*}$ on $M_{2 \kappa}$, so $M_{2 \kappa}$ contains all the information about $Y_{\kappa}^{*}$.

Theorem 1. Let $Y_{\kappa}=\left[\Delta^{2 \kappa+2}\right]^{\kappa}$. Then

$$
\text { index } Y_{\kappa}^{*}=2 \kappa \text {. }
$$

Proof. It suffices to prove that index $M_{2 \kappa}=2 \kappa$. As follows from Lemma 1, there exists in $\mathbb{R}^{2 \kappa}$ a set

$$
M=\left\{a_{1}, a_{2}, \ldots, a_{2 \kappa+3}\right\}
$$

of $2 \kappa+3$ points such that $\#_{\kappa}(M)=1$. Let the single $\kappa$-intersection arise between the simplexes $\left[a_{1}, \ldots, a_{\kappa+1}\right]$ and $\left[a_{\kappa+2}, \ldots, a_{2 \kappa+2}\right]$. Consider in $\mathbb{R}^{2 \kappa+1}$ the set

$$
N=\left\{a_{1}+l_{2 \kappa+1}, a_{2}, \ldots, a_{2 \kappa+3}\right\},
$$


where $l_{2 \kappa+1}$ is a unit vector orthogonal to $\mathbb{R}^{2 \kappa}$. Clearly, $\#_{\kappa}(N)=0$. Then $Y_{\kappa}=$ $\left[\Delta^{2 \kappa+2}\right]^{\kappa}$ may be embedded in $\mathbb{R}^{2 \kappa+1}$ with vertices in $N$. Let us define an odd map $\varphi: M_{2 \kappa} \rightarrow S^{2 \kappa}$ as follows:

$$
\varphi(x, y)=\frac{y-x}{\|y-x\|} .
$$

The preimage $\varphi^{-1}\left(-l_{2 \kappa+1}\right)$ contains a single point $\left(x_{0}, y_{0}\right)$ corresponding to the single $\kappa$-intersection in $M$. Here $x_{0} \in\left[a_{1}+l_{2 \kappa+1}, \ldots, a_{\kappa+1}\right], y_{0} \in\left[a_{\kappa+2}, \ldots, a_{2 \kappa+2}\right]$. We shall prove that $\operatorname{deg}_{2} \varphi=1(\bmod 2)$. It is clear that there exists in $S^{2 \kappa}$ a neighbourhood $W \ni-l_{2 \kappa+1}$ such that the map $\varphi$ restricted to $\varphi^{-1}(W)$ is a homeomorphism. Let us approximate $\varphi$ with a smooth map $\varphi_{0}: M_{2 \kappa} \rightarrow S^{2 \kappa}$ such that $\varphi_{0}(x)=\varphi(x)$ for $x \in \varphi^{-1}(W)$. Then $-l_{2 \kappa+1}$ is a regular value of $\varphi_{0}$ and $\varphi_{0}^{-1}\left(-l_{2 \kappa+1}\right)$ contains a single point $\left(x_{0}, y_{0}\right)$. But then for its degree mod 2 we have $\operatorname{deg}_{2} \varphi_{0}=1$ (cf. [2]), and therefore $\operatorname{deg}_{2} \varphi=1$.

Then index $M_{2 \kappa}=2 \kappa$, as follows from the Proposition in the preliminary section.

The theorem is proved.

Note that if $P_{\kappa}$ is a contractible $\kappa$-dimensional polyhedron, then index $P_{\kappa}^{*} \leq$ $2 \kappa-1$. This is established in [3].

Theorem 2. For any $\kappa \geq 1$ there exists a closed manifold $M_{2 \kappa}$ with a free involution $T$, such that index $M_{2 \kappa}=2 \kappa$, but there is a map

$$
f: M_{2 \kappa} \rightarrow Y_{\kappa}
$$

into a $\kappa$-dimensional polyhedron without antipodal coincidence: $f(T x) \neq f(x)$ for any $x \in M_{2 \kappa}$.

Proof. Let $M_{2 \kappa}$ and $Y_{\kappa}$ be as in Theorem 1. Set $f(x, y)=x$. Then $f: M_{2 \kappa} \rightarrow Y_{\kappa}$ is a map without antipodal coincidence: $f(x, y) \neq f(y, x)$.

Let us note that not every manifold of index $2 \kappa$ admits such a map. Šchepin [4] has shown that every map $f: S^{2 \kappa} \rightarrow P_{\kappa}$ of the sphere $S^{2 \kappa}$ into a $\kappa$-dimensional polyhedron has an antipodal coincidence: $f(-x)=f(x)$.

The following is a simple but useful proposition that we shall refer to in the last two sections.

Proposition $(*)$. Let $\Delta^{n}=\left[a_{1}, \ldots, a_{n+1}\right]$ be the standard $n$-simplex in $\mathbb{R}^{n}$. Suppose that $\sum_{i=1}^{n+1} \lambda_{i} a_{i}=0$. Then $\lambda_{1}=\lambda_{2}=\cdots=\lambda_{n+1}$.

Proof. Since $\sum a_{i}=0$, we find $a_{1}=-a_{2}-\cdots-a_{n+1}$. Substitute in $\sum \lambda_{i} a_{i}=0$ and make use of the fact that $a_{2}, \ldots, a_{n+1}$ are independent.

\section{Proof that $M_{2 \kappa}$ IS A MANIFOLD}

In this section we shall prove Lemma 2. The key is Lemma 3, which is interesting for itself. Let

$$
P_{\kappa}=O\left(\left[\Delta^{2 \kappa}\right]^{\kappa-1}\right)
$$

be the cone over $\left[\Delta^{2 \kappa}\right]^{\kappa-1}$ with vertex the origin $O$. Consider the set

$$
U=\left\{(x, y) \in P_{\kappa}^{2} \mid[x] \cap[y]=\{O\}\right\} .
$$


Lemma 3. The map $\varphi: U \rightarrow \mathbb{R}^{2 \kappa}$ defined by

$$
\varphi(x, y)=x-y
$$

maps $U$ homeomorphically onto some open star-like subset of $\mathbb{R}^{2 \kappa}$ with center $O$.

Proof. Let $\Delta^{2 \kappa}=\left[a_{1}, \ldots, a_{2 \kappa+1}\right]$. If $(x, y) \in U$, then

$$
x=\sum_{i \in I} \alpha_{i} a_{i}, \quad y \in \sum_{j \in J} \beta_{j} a_{j},
$$

where $\alpha_{i}, \beta_{j}>0$ and $|I| \leq \kappa,|J| \leq \kappa, I \cap J=\varnothing$. (The index sets are disjoint, since $[x] \cap[y]=\{O\}$ by definition.)

1) First we prove that $\varphi$ is "mono".

Let $\varphi(x, y)=\varphi(u, v)$, i.e. $x-y=u-v$. We have as above

$$
u=\sum_{r \in R} \gamma_{r} a_{r}, \quad v=\sum_{s \in S} \delta_{s} a_{s},
$$

where $\gamma_{r}, \delta_{s}>0,|R| \leq \kappa,|S| \leq \kappa, R \cap S=\varnothing$. Then

$$
\sum_{I} \alpha_{i} a_{i}-\sum_{J} \beta_{j} a_{j}-\sum_{R} \gamma_{r} a_{r}+\sum_{S} \delta_{s} a_{s}=0 .
$$

Let

$$
M=\{1,2, \ldots, 2 \kappa+1\} .
$$

By Proposition $(*)$ all the coefficients in (2) are (after reduction) equal to some number $c$.

If $c \geq 0$, then from (2) $J \cup R$ is contained in $I \cup S$, so $I \supset R$ and $S \supset J$. Since $|S|+|I| \leq 2 \kappa<|M|$, this implies $I \cup J \cup R \cup S \neq M$, thus $c=0$.

If $c \leq 0$, then in the same way it follows that $R \supset I, J \supset S$ and $I \cup J \cup R \cup S \neq M$, hence $c=0$.

The single possibility for this is $I=R, J=S$. But then (2) implies that

$$
\sum_{I} \alpha_{i} a_{i}=\sum_{R} \gamma_{r} a_{r}, \quad \sum_{J} \beta_{j} a_{j}=\sum_{S} \delta_{s} a_{s} .
$$

So, $x=u, y=v$, i.e. $\varphi$ is "mono".

2) We shall show that $\varphi(U)$ contains $O$ in its interior.

Let $w \in \mathbb{R}^{2 \kappa}$ be a vector with a small norm. Then it may be written in the form

$$
w=\lambda_{1} a_{1}+\lambda_{2} a_{2}+\cdots+\lambda_{2 \kappa} a_{2 \kappa},
$$

where we assume that $\lambda_{1} \leq \lambda_{2} \leq \cdots \leq \lambda_{2 \kappa}$.

Clearly,

$$
a_{\kappa}=-a_{1}-\cdots-a_{\kappa-1}-a_{\kappa+1}-\cdots-a_{2 \kappa+1}
$$

and, substituting above,

$$
\begin{aligned}
w= & \left(\lambda_{1}-\lambda_{\kappa}\right) a_{1}+\cdots+\left(\lambda_{\kappa-1}-\lambda_{\kappa}\right) a_{\kappa-1} \\
& +\left(\lambda_{\kappa+1}-\lambda_{\kappa}\right) a_{\kappa+1}+\cdots+\left(\lambda_{2 \kappa}-\lambda_{\kappa}\right) a_{2 \kappa}-\lambda_{\kappa} a_{2 \kappa+1} .
\end{aligned}
$$

Set

$$
\begin{gathered}
x=\left(\lambda_{\kappa+1}-\lambda_{\kappa}\right) a_{\kappa+1}+\cdots+\left(\lambda_{2 \kappa}-\lambda_{\kappa}\right) a_{2 \kappa}, \\
y=\left(\lambda_{\kappa}-\lambda_{1}\right) a_{1}+\cdots+\left(\lambda_{\kappa}-\lambda_{\kappa-1}\right) a_{\kappa-1}+\lambda_{\kappa} a_{2 \kappa+1} .
\end{gathered}
$$

Then $w=x-y$, where $x, y \in O\left(\left[\Delta^{2 \kappa}\right]^{\kappa-1}\right)$, since $x$ and $y$ are of small norm and the coefficients are nonnegative. Note also that $[x] \cap[y]=\{O\}$, thus $(x, y) \in U$. 
So $w=\varphi(x, y)$, hence $w \in \varphi(U)$. We proved that $\varphi(U)$ contains $O$ in its interior. Note finally, that $\varphi(U)$ is star-like, as follows immediately from the definition of $\varphi$.

The lemma is proved.

Proof of Lemma 2. Here we prove that $M_{2 \kappa}$ is a manifold. Recall that

$$
M_{2 \kappa}=\left\{(x, y) \in Y_{\kappa}^{2} \mid[x] \cap[y] \neq \varnothing\right\},
$$

where $Y_{\kappa}=\left[\Delta^{2 \kappa+2}\right]^{\kappa}$. It is clear that $M_{2 \kappa}$ is subdivided in a natural way to prisms of the form $\sigma^{i} \times \sigma^{j}$, where $\sigma^{i}, \sigma^{j} \subset Y_{\kappa}$ are nonintersecting simplexes. We shall prove that the star of each vertex of $M_{2 \kappa}$ is homeomorphic to $\mathbb{R}^{2 \kappa}$. It suffices to check it for an arbitrary vertex $\left(a_{1}, a_{2}\right)$. Clearly,

$$
\operatorname{St}\left(a_{1}, a_{2}\right)=\left\{(x, y) \in Y_{\kappa}^{2} \mid[x] \ni a_{1},[y] \ni a_{2},[x] \cap[y]=\varnothing\right\} .
$$

We shall embed $Y_{\kappa}$ in $\mathbb{R}^{2 \kappa+1}$ as follows: Let $\left\{a_{3}, \ldots, a_{2 \kappa+3}\right\}$ be the vertices of the standard simplex $\Delta^{2 \kappa}$ in $\mathbb{R}^{2 \kappa}$ and $a_{1}=l_{2 \kappa+1}, a_{2}=-l_{2 \kappa+1}$, where $l_{2 \kappa+1}$ is a unit vector orthogonal to $\mathbb{R}^{2 \kappa}$. The points $\left\{a_{1}, \ldots, a_{2 \kappa+3}\right\}$ are in general position in $\mathbb{R}^{2 \kappa+1}$, consequently we may embed there $Y_{\kappa}=\left[\Delta^{2 \kappa+2}\right]^{\kappa}$ with vertices $a_{i}$. Let $p: \mathbb{R}^{2 \kappa+1} \rightarrow \mathbb{R}^{2 \kappa}$ denote the orthogonal projection. We have

$$
p\left(Y_{\kappa}\right)=O\left(\left[\Delta^{2 \kappa}\right]^{\kappa-1}\right) .
$$

It is easy to see that $(x, y) \in \operatorname{St}\left(a_{1}, a_{2}\right)$ if and only if $(p(x), p(y)) \in U$, where $U$ is defined by (1). Then the map $P(x, y)=(p(x), p(y))$ is a homeomorphism and according to Lemma $3, U$ is homeomorphic to $\mathbb{R}^{2 \kappa}$. Therefore $\operatorname{St}\left(a_{1}, a_{2}\right) \approx \mathbb{R}^{2 \kappa}$.

\section{Proof of Lemma 1}

Here we shall construct in $\mathbb{R}^{2 \kappa}$ a set $M$ of $2 \kappa+3$ points such that $\#_{\kappa}(M)=1$. Let $\Delta^{2 \kappa}=\left[a_{1}, \ldots, a_{2 \kappa+1}\right]$ be the standard simplex in $\mathbb{R}^{2 \kappa}$. Set

$$
A=\lambda\left(a_{1}+\cdots+a_{\kappa}\right)+\delta a_{\kappa+1},
$$

where $0<\delta<\lambda$ and $\kappa \lambda+\delta<1$.

We shall show that the set

$$
M=\left\{O, a_{1}, \ldots, a_{2 \kappa+1}, A\right\}
$$

meets the case. Note first that $A$ lies in the interior of $\Delta^{2 \kappa}$, since $\kappa \lambda+\delta<1$. Consider two $\kappa$-dimensional simplexes $\sigma_{1}$ and $\sigma_{2}$ with vertices in $M$ without a common vertex. If some of them contain neither $O$, nor $A$, then $\sigma_{1} \cap \sigma_{2}=\varnothing$. Indeed, suppose that $O, A \notin \sigma_{1}$. Then $\sigma_{1} \subset \partial \Delta^{2 \kappa}$ and $\sigma_{1} \cap \sigma_{2}=\sigma_{1} \cap\left(\sigma_{2} \cap \partial \Delta^{2 \kappa}\right)=\varnothing$. The last equality holds, since $\sigma_{2} \cap \partial \Delta^{2 \kappa}$ is a simplex in $\partial \Delta^{2 \kappa}$ without common vertices with $\sigma_{1}$.

So, we may suppose that

$$
\sigma_{1}=\left[O, a_{i_{1}}, \ldots, a_{i_{\kappa}}\right], \quad \sigma_{2}=\left[A, a_{j_{1}}, \ldots, a_{j_{\kappa}}\right],
$$

where all the vertices are different. Suppose that $\sigma_{1}$ and $\sigma_{2}$ intersect; then we have

$$
\lambda_{1} a_{i_{1}}+\cdots+\lambda_{\kappa} a_{i_{\kappa}}=\mu_{0} \lambda\left(a_{1}+\cdots+a_{\kappa}\right)+\mu_{0} \delta a_{\kappa+1}+\mu_{1} a_{j_{1}}+\cdots+\mu_{\kappa} a_{j_{\kappa}},
$$

where $\lambda_{i} \geq 0, \sum \lambda_{i} \leq 1, \mu_{j} \geq 0, \sum \mu_{j}=1$.

Note that $\mu_{0}>0$, since $\sigma_{1}$ and $\sigma_{2}$ may intersect only in an interior point of $\Delta^{2 \kappa}$.

Send all the members of (3) to the right-hand side, then each $a_{i}$ appears (after reduction) with some coefficient $\nu\left(a_{i}\right)$. According to Proposition $(*)$ we should have

$$
\nu\left(a_{1}\right)=\nu\left(a_{2}\right)=\cdots=\nu\left(a_{2 \kappa+1}\right) .
$$


Let us note that exactly one $a_{i}$ is not a vertex of either $\sigma_{1}$ or of $\sigma_{2}$. There are 3 possibilities about $a_{i}$.

1) $i \geq \kappa+2$. Then $\nu\left(a_{i}\right)=0$ and hence $\nu\left(a_{j}\right)=0$ for any $j$. But there exists some $j \leq \kappa+1$ such that $a_{j}$ is a vertex of $\sigma_{2}$. Then the examination of (3) gives $\nu\left(a_{j}\right)>0$, in contradiction with (4).

2) $i \leq \kappa$. Then $\nu\left(a_{i}\right)=\mu_{0} \lambda>0$. This implies that for $j \geq \kappa+2$ all the $a_{j}$ are in the right-hand side of $(3)$, otherwise we would have $\nu\left(a_{j}\right) \leq 0$, in contradiction with (4). Then $a_{\kappa+1}$ takes part in the left-hand side with coefficient $\lambda_{\kappa+1} \geq 0$. Therefore

$$
\nu\left(a_{\kappa+1}\right)=\mu_{0} \delta-\lambda_{\kappa+1}=\nu\left(a_{i}\right)=\mu_{0} \lambda,
$$

so $\mu_{0}(\delta-\lambda)=\lambda_{\kappa+1} \geq 0$, which contradicts the condition $\delta<\lambda$.

3) $i=\kappa+1$, i.e. $a_{\kappa+1}$ is not a vertex of $\sigma_{1} \cup \sigma_{2}$. In this case we should have

$$
\sigma_{1}=\left[O, a_{1}, \ldots, a_{\kappa}\right], \quad \sigma_{2}=\left[A, a_{\kappa+2}, \ldots, a_{2 \kappa+1}\right] .
$$

Really, if we suppose that $a_{j} \in \sigma_{1}$ for some $j \geq \kappa+2$, then $a_{j}$ takes part in the left-hand side of (3) with coefficient $\lambda_{j} \geq 0$, thus $\nu\left(a_{j}\right)=-\lambda_{j}$, though $\nu\left(a_{\kappa+1}\right)=\mu_{0} \delta>0$, in contradiction with (4).

We shall prove that $\sigma_{1}$ and $\sigma_{2}$ intersect in an interior point. We are looking for a solution of

$$
\begin{aligned}
\lambda_{1} a_{1}+\cdots+\lambda_{\kappa} a_{\kappa}= & \mu_{0} \lambda\left(a_{1}+\cdots+a_{\kappa}\right)+\mu_{0} \delta a_{\kappa+1} \\
& +\mu_{\kappa+2} a_{\kappa+2}+\cdots+\mu_{2 \kappa+1} a_{2 \kappa+1}
\end{aligned}
$$

with $\lambda_{i}, \mu_{j} \geq 0, \sum \lambda_{i} \leq 1, \sum \mu_{j}=1$.

It is straightforward to check that the numbers

$$
\begin{gathered}
\lambda_{1}=\lambda_{2}=\cdots=\lambda_{\kappa}=\frac{\lambda-\delta}{1+\delta \kappa}, \quad \mu_{0}=\frac{1}{1+\delta \kappa}, \\
\mu_{\kappa+2}=\cdots=\mu_{2 \kappa+1}=\frac{\delta}{1+\delta \kappa}
\end{gathered}
$$

satisfy (5), since it reduces to the identity $\sum a_{i}=0$. This means that $\sigma_{1}$ and $\sigma_{2}$ intersect in an interior point. Therefore $\#_{\kappa}(M)=1$.

\section{CONCLUDing REMARKS}

There is a natural generalization of the spaces $Y_{\kappa}$. Let as above $Y_{\kappa}=\left[\Delta^{2 \kappa+2}\right]^{\kappa}$ and consider the join

$$
X_{\kappa}=Y_{\kappa_{1}} * Y_{\kappa_{2}} * \cdots * Y_{\kappa_{p}}
$$

where $\kappa=\kappa_{1}+\cdots+\kappa_{p}+p-1$. It is not difficult to see that the spaces $X_{\kappa}$ also have index $X_{\kappa}^{*}=2 \kappa$. It is shown in [1] that $X_{\kappa}$ is a $\kappa$-minimal complex, in the sense that it is not embeddable in $\mathbb{R}^{2 \kappa}$ but each of its proper subcomplexes is embeddable in $\mathbb{R}^{2 \kappa}$. Cohomological obstructions for embedding in $\mathbb{R}^{2 \kappa}$ are discussed in [5] and [6].

For a graph $\Gamma$ the question of computing index $\Gamma^{*}$ is in fact solved in [6]:

We have index $\Gamma^{*}=2$ if and only if $\Gamma$ is nonplanar. Furthermore, index $\Gamma^{*}=1$ if and only if $\Gamma$ is planar, but not embeddable in $\mathbb{R}^{1}$. Otherwise index $\Gamma^{*}=0$.

Another interesting phenomenon in the case of graphs is the following fact. Let

$$
M(\Gamma)=\left\{(x, y) \in \Gamma^{2} \mid[x] \cap[y]=\varnothing\right\} .
$$

Then $M(\Gamma)$ is homeomorphic to a closed surface if and only if $\Gamma$ is one of the two Kuratowski graphs $K_{5}$ and $K_{3,3}$. Moreover, $M\left(K_{5}\right)$ is a sphere with 6 handles and 
$M\left(K_{3,3}\right)$ is a sphere with 4 handles. This can be shown by computing the Euler characteristic of these spaces and by checking that they are both orientable.

I thank the referee for pointing my attention to papers [5] and [6].

\section{REFERENCES}

1. B. Grünbaum, Imbeddings of simplicial complexes, Comment. Math. Helvet. 44 (1969), 502513. MR 40:8058

2. M. W. Hirsch, Differential Topology, Springer-Verlag, New York, 1976. MR 56:6669; MR 96c:57001, corrected reprint

3. M. Izydorek, J. Jaworowski, Antipodal coincidence for maps of spheres into complexes, PAMS 123 (1995), 1947-1950. MR 96c:55002

4. E. V. Šchepin, On a problem of L. A. Tumarkin, Dokl. Akad. Nauk SSSR 217 (1974), 42-43.

5. B. R. Ummel, Imbedding classes and n-minimal complexes, PAMS 38 (1973), 201-206. MR 47:5883

6. W. T. Wu, A theory of imbedding, immersion and isotopy of polytopes in a euclidean space, Science Press, Peking, 1965.

7. C. T. Yang, On theorems of Borsuk-Ulam, Kakutani-Yamabe-Yujobô and Dyson, I, Ann. Math. 60 (1954), 262-282. MR 16:502d

1 Suchodolska Str., B 13 Vh 2 Ap 32, 1373 Sofia, Bulgaria

E-mail address: s_simeon@hotmail.com 